\title{
Do readers make inferences about conversational topics?
}

\author{
R. BROOKE LEA and PATRICK A. KAYSER \\ Macalester College, St. Paul, Minnesota \\ ELIZABETH J. MULLIGAN \\ University of Colorado, Boulder, Colorado \\ and \\ JEROME L. MYERS \\ University of Massachusetts, Amherst, Massachusetts
}

\begin{abstract}
When we read that two protagonists in a story chatted together for a couple of minutes, do we draw inferences about the topic of the conversation on the basis of information presented earlier in the text? Participants read passages in which protagonists part and later reunite; the passages ended with a sentence either that implied conversation or did not. In Experiment 1, participants' continuation sentences indicated that inferences about the topic of conversation were drawn. Recognition probe data in Experiment 2 provided more immediate evidence of such inferences. Experiment 3 addressed a possible confound in Experiment 2 and again provided evidence that readers inferred the continuation of the conversation. In Experiments 4 and 5, we investigated the effect of having the targeted conversational topic be a secret that should not be shared between the protagonists. The results are discussed in terms of the collaboration between passive, memory-based text processing and schema-driven comprehension processes.
\end{abstract}

It is well established that readers draw inferences during reading when they are necessary to maintain the referential or causal coherence of a text (e.g., Garrod \& Sanford, 1977; Haviland \& Clark, 1974; Keenan, Baillet, \& Brown, 1984; McKoon \& Ratcliff, 1980; Myers, Shinjo, \& Duffy, 1987; O’Brien, Duffy, \& Myers, 1986; Potts, Keenan, \& Golding, 1988). ${ }^{1}$ There has been less consensus about the status of inferences that are not necessary to maintain local coherence. One type of non-necessary inference, called "elaborative inference," merely expands upon some aspect of the text in a way that is optional with regard to the coherence of the text. For example, consider the following sentence pair (Potts et al., 1988):

1. No longer able to control his anger, the husband threw the delicate porcelain vase against the wall.

2. It cost him well over one hundred dollars to replace the vase.

A bridging inference (that the vase broke) is needed to coherently link Sentences 1 and 2 . Without that inference,

This research was supported by a Keck Foundation Grant from Macalester College to R.B.L. and P.A.K. We thank Ira Noveck, Barry Cohen, Akiva Liberman, and Anne Cook for helpful conversations and suggestions regarding this research. We also thank Hannah Jackson, Hilary Mead, Marc Johansen, Jesse Calm, and Terri Ann Thompson for assistance with data collection and coding. Correspondence should be addressed to R. B. Lea, Department of Psychology, Macalester College, 1600 Grand Ave., Saint Paul, MN 55105 (e-mail: lea@ macalester.edu). readers would not understand why the husband had to buy a new vase. In contrast, no inference is required to maintain coherence when Sentence 1 is followed by the following sentence:

2a. He had been feeling angry for weeks, but had refused to seek help.

If readers infer that the vase broke, they would be making a particular form of elaborative inference-a predictive inference, anticipating an event that is not explicitly mentioned in the text and is not needed to maintain coherence in the text.

The results of many of the earliest studies of predictive inferences indicated that readers make inferences while reading to maintain coherence but that they do not routinely make predictive inferences (e.g., McKoon \& Ratcliff, 1986; Potts et al., 1988; Singer, 1979; Singer \& Ferreira, 1983). Such results are consistent with the assumption that predictive inferences are not made by readers because there are too many possible continuations of a narrative to allow for a correct prediction and because prediction would, in many instances, require considerable working memory resources. However, the results of more recent experiments (Calvo \& Castillo, 1996; Calvo, Castillo, \& Estevez, 1999; Estevez \& Calvo, 2000; Keefe \& McDaniel, 1993; Klin, Murray, Levine, \& Guzman, 1999; Murray, Klin, \& Myers, 1993) have demonstrated that predictive inferences do occur during reading. These results indicate that at least one form of elaborative inference, predictive inference, is made under some circumstances. 
Demonstrations of predictiveinferences may be accounted for by relations between the explicit text and the reader's general world knowledge. Kintsch's (1988) constructionintegration (C-I) model provides one account of how relations between the text and the reader's knowledge can result in a predictive inference. Kintsch assumes that in the construction phase the text is first parsed into concepts and propositions. These then serve as retrieval cues for associated concepts and propositions in the network of knowledge the reader brings to the text. This dumb, bottomup, process results in an "initial, enriched, but incoherent and possibly contradictory text base" (Kintsch, 1988, p. 166). These incoherencies and contradictions are then resolved in an integration phase by a constraint-satisfaction process. The $\mathrm{C}-\mathrm{I}$ model can provide an account of the predictive inferences demonstrated in previous studies if it is assumed that The angry husband threw the vase against the wall initiates a construction phase that activates propositions like fragile vases sometimes break and throwing such objects causes them to break. The integration phase would then result in the addition of the proposition that the vase broke to the discourse representation.

It is less clear whether elaborative inferences are ordinarily drawn that do not depend on activation of related concepts and propositions in the reader's knowledge base. However, some evidence that there are circumstances in which such inferences occur is provided by the results of experiments by Lea (1995) showing that readers draw logical inferences that are not needed for coherence. For example, a passage might contain the information that the dieting protagonist could have either bread or cereal for breakfast, and that the protagonist decided not to have the cereal. The logical inference is that the protagonist selected the bread. Participants named butter, a semantic associate of bread, faster following this passage than following a no-inference control, indicating that the inference had been drawn. In contrast to experiments that demonstrated predictive inferences (e.g., Keefe \& McDaniel, 1993; Murray et al., 1993), the activation and integration of propositions in the reader's knowledge base is not sufficient to account for Lea's results (see also Lea \& Mulligan, 2002). Drawing an analogy to Kintsch's (1988) discussion of how the C-I model would deal with part-whole schemas in the solution of arithmetic word problems, Lea suggested that an abstract propositional-logicschema was activated in his experiments.

In the present set of experiments, we consider another paradigm in which inferences appear to require more than the activation and integration of related concepts and propositions in the reader's knowledge base. In these experiments, we investigated whether readers make elaborative inferences about the topic of a conversation between two protagonists. Table A1 presents four versions of a passage in which two protagonists part and then reunite; the standard conclusion portion was taken from previous studies (e.g., Lea, Mason, Albrecht, Birch, \& Myers, 1998), and the three discussion conclusions were used in Experiment 1 . The results of previous studies (Greene, Gerrig,
McKoon, \& Ratcliff, 1994; Lea et al., 1998; McKoon, Gerrig, \& Greene, 1996) have shown that reinstating Jane reactivates a concept (cousin) associated with her. That is, cousin was inactive before the reunion sentence but was reactivated following that sentence. The question the present experiments address is whether, following a sentence in which the protagonists are talking, the reader will infer that Jane's cousin will be the topic of their discussion. For example, consider the discussion conclusion to the passage in Table A1. After Gloria and Jane reunited, the discussion sentence says, They chatted together for a couple of minutes. Will readers infer that they are talking about the cousin? What if their conversation is initiated with more urgency (see urgent-discussion conclusion): "I have got to tell you something," said Jane?

The question is of interest because inferences about the topic of discussion would appear to run counter to the generally accepted position that elaborative inferences are infrequent (e.g., Graesser, Singer, \& Trabasso, 1994; McKoon \& Ratcliff, 1992, 1995), and inferences about the topic of discussion seem quite different from the associative inferences demonstrated in studies in which there is a strong prior relation between text propositions and general knowledge propositions (e.g., Keefe \& McDaniel, 1993; Murray et al., 1993). In the discussion paradigm, there is no prior relation between the focal (the discussion) sentence and the targeted inference (the protagonists will discuss the cousin). Nor is there a logical relation between the discussion sentence and the inference, as there was between the premises in Lea's (1995) experiments and the inference. Thus, the inference does not appear to be strongly constrained by the context; although cousin has been reactivated by the reunion, it has not been recently mentioned and other topics could be introduced without violating the coherence of the text.

Despite these considerations, there are grounds for hypothesizing that the urgent-discussion condition, or both discussion conditions, will yield evidence that readers infer that the topic of conversation is the targeted concept (e.g., Jane's cousin). The urgent-discussion sentence may serve to motivate the reader to consciously anticipate the continuation of the narrative. Because the reunion sentence has reactivated the cousin, the reader may select that as the topic of the discussion. Alternatively, phrases such as They chatted together may activate a conversation schema containing slots for the discussants and the conversational topic. The topic slot would be filled with information that was readily available, presumably the concept (e.g., cousin) that had been reactivated by the reunion sentence. Under this hypothesis, there should be evidence of an inference in both discussion conditions.

In the present experiments, Lea et al.'s (1998) texts were modified to test whether a potential topic of discussion, such as cousin, would be inferred. In Experiment 1, participants read the passages up to the discussion sentence and were instructed to write a few lines to finish the story. Numbers of mentions of the backgrounded concept were compared across conditions. Experiment 2 used recogni- 
tion probes following the discussion sentence to test whether cousin would be more active following the discussion or urgent-discussion sentences than following the no-discussion sentence. Experiment 3 addressed the possibility that differences among reunion sentences, rather than differences among discussion sentences, accounted for effects obtained in Experiments 1 and 2. Experiment 4 replicated Experiment 2 with the addition of a condition in which one protagonistkeeps a secret from the other (e.g., a surprise party one protagonist is planning for the other). To the extent that conscious top-down processes play a role in the inferential process, there should be evidence that the reader hesitates in inferring that the secret is the topic of conversation. Experiment 5 was a continuationwriting version of Experiment 4 that helped us interpret the urgent-discussion results in the previous experiment.

\section{EXPERIMENT 1}

Experiment 1 investigated whether readers would infer that continuations of the story would include the target concept. Participants were given versions of the experimental stories to read that ended at the discussion sentence and they were asked to write a continuation of the story. Their endings were then coded with regard to whether or not the target character was mentioned. If readers anticipate that the topic of conversation will be the target concept, the number of target character mentions should be greater for the discussion sentence conditions than for the control condition. The lack of a general knowledge-based connection between the concept and the discussion sentence, together with the fact that there are many possible ways in which the narrative could continue, argue against such an effect. Nevertheless, Lea's (1995) evidence that logical inferences are made suggests at least the possibility that other elaborative inferences may occur during reading.

\section{Method}

Participants. Eighteen Macalester College students participated in Experiment 1 in return for course credit. All participants were native speakers of English.

Materials. Thirty-six of the 42 stories used by Lea et al. (1998) were selected at random as materials for this experiment. These texts were based on those used by Greene et al. (1994). An example of the texts used in Experiment 1 was presented in Table A1. Each passage ranged in length from 13 to 16 sentences and was composed of three sections: introduction, continuation, and conclusion. The introduction section, which was 4-6 sentences long, introduced the two main protagonists of the story. The protagonists, who were designated with proper names, then parted and a third, target character, was introduced into the context of one of the protagonists. The target character was referred to by social role (e.g., cousin, professor) and occasionally by proper name (e.g., Marilyn, Senator Bigelow). In the example passage (see Table A1), Jane and Gloria were introduced and then parted before Jane's cousin, Marilyn, was introduced in the context of Jane going to have dinner with her. In the continuation section, which was 5-6 sentences long, the story follows the actions of the protagonists that were not associated with the target character; no mention was made of the other protagonist or target character. In the example passage, Gloria's evening was described with no mention of Jane or her cousin. There were three different versions of the con- clusion section. Two of the conclusion sections contained the experimentally manipulated discussion sentences; after the two protagonists were reunited in the story, a conversation was initiated with the use of either an urgent-discussion sentence like I have got to tell you something or an ordinary discussion sentence like They chatted together for a couple of minutes. The no-discussion conclusion, which acted as a control for the discussion sentence manipulations, contained a no-discussion sentence. In the latter condition the protagonists were reunited in a different manner in order to clearly establish that they would not converse. In the example story, Gloria was sound asleep when Jane arrived home, making discussion impossible.

The 36 passages were divided into three stimulus lists each with 12 passages in each of the three conditions (discussion, urgent discussion, and no discussion). Thus, three stimulus lists were created, each with 36 passages. The stimulus lists were printed in paper packets, one story on a page. The stories were printed in black ink on white $8 \times 11$ in. sheets of paper. The stories ended after the discussion sentence. Immediately following the discussion sentence three blank lines were provided on which the participants could write their continuations. The experimental conditions in this and all subsequent experiments were within subjects.

Procedure. Each participant was randomly assigned to read one of the three stimulus packets. All materials were presented in packet form to the participants. Participants were instructed to read each story at a comfortable pace and write how they thought each story would finish in the blank lines provided at the end of the stories. Participants were told to try to complete the stories in ways that were congruent with the rest of the story. The experimental sessions lasted approximately $1 \mathrm{~h}$.

\section{Results and Discussion}

Two criteria were developed to operationally define a mention of the target character. By the "reference" criterion, any unambiguous reference to the target was counted as a mention. For the passage presented in Table A1, for example, any continuation that included cousin, Marilyn, or she/her when the pronoun unambiguously referred to the cousin was coded as mentioning the target. By the "nominal" criterion, only proper names (Marilyn) or social roles (cousin) were counted as mentions. Two experimenters coded the participants' protocols using both the reference and nominal criteria. No statistical difference was found between the two mention categories. Therefore the more inclusive reference criterion was used for analysis.

Results reported as significant had associated $p$ values of less than .05. On average, the target was mentioned in the no-discussion control 5.06 times in the 12 opportunities to do so (42\%), 7.94 times in the discussion condition $(66 \%)$, and 10 times in the urgent-discussion condition (83\%). This effect of the discussion condition was significant $\left[F(2,34)=57.31, M S_{\mathrm{e}}=1.938\right]$. Two-tailed $t$ tests were conducted among the means of the three conditions. The discussion versus no-discussion comparison showed a significant difference in the proportion of participants who mentioned the concept of the target character $[t(17)=$ $5.66, S E M=.5106]$. Likewise, the urgent-discussion versus no-discussion comparison produced a significant difference $[t(17)=10.73, S E M=.4607]$. Participants on average mentioned the target protagonist more after urgentdiscussion passages than after discussion passages $[t(17)=$ $4.94, S E M=.4160]$. Thus, all three conditions were significantly different from each other. 
The significant differences found between the nodiscussion condition and the two experimental conditions provide preliminary evidence of an elaborative inference following discussion sentences. In addition, the urgentdiscussion condition resulted in a significantly higher proportion of concept mentions on average than did the discussion condition, indicating a greater probability of an inference when the narrative stresses the importance of the topic in the forthcoming discussion. In summary, although the targeted conversational topic had been backgrounded by text intervening between it and the discussion sentence, readers did mention it, and the number of such mentions depended upon the type of discussion sentence. However, Experiment 1 demonstrated these effects only when participants were required to consciously construct a continuation of the story. The next three experiments test whether such inferential effects occur spontaneously.

\section{EXPERIMENT 2}

In Experiment 1 we found that readers were much more likely to write continuations containing the target character when the stories ended in conversation than when the protagonists were not talking. This result suggests that (1) the target character was sufficiently available to be included in the conclusions readers wrote for the stories, and (2) the target character was predictably related to what happened next in the stories when the stories ended with a discussion. In Experiment 2 we returned to the on-line methodology used in previous research, which features the present set of materials (i.e., Greene et al., 1994; Lea et al., 1998; McKoon et al., 1996) to look for evidence that readers make elaborative inferences about the topics of discussions even when they are not asked to. That is, we wondered whether the targeted concept would be more active after discussion and urgent-discussion sentences than after the no-discussion sentence.

\section{Method}

Participants. Forty-five Macalester College students participated in Experiment 2 in return for course credit. All participants were native speakers of English.

Materials. The 42 experimental passages consisted of the 36 texts used in Experiment 1, plus the 6 additional passages from the original 42 used by Lea et al. (1998) and Greene et al. (1994). See the Materials section of Experiment 1 for a complete description of the materials. An example text is presented in Table A1. Immediately following the discussion sentence, a recognition test word that referred to the target character was presented in each conclusion section. The recognition test word was followed by two concluding sentences. The pre-probe lines were equated for length to minimize the chance that differential line length might affect the recognition times. The mean pre-probe line length was $38.9,40.1$, and 40.7 characters for the no-discussion, discussion, and urgent-discussion passages, respectively.

The 26 filler passages were taken from Lea et al. (1998), which in turn had been taken from Greene et al. (1994). They varied in length from 8 to 13 sentences. Their style was similar to that of the experimental items in that they described two or more characters that interacted. However, the characters interacted with each other differently than in the experimental items. For example, in approxi- mately half the filler passages, the characters did not separate from each other. The filler passages were also different from the experimental items in that they contained three probe words for each story. Using three probe words per story helped obscure patterns in the experimental trials by introducing negative trials, especially toward the end of the story, and by probing in all parts of the passages. Correct responses in the first two thirds of the filler stories were half positive and half negative, and those in the final third were all negative. The probe words for the filler passages were common nouns with approximately one half of the negative test words referring to social roles. Overall, 78 of the recognition task responses were to filler probes and 42 were to experimental probes.

Procedure. Each participant completed a session that lasted approximately $1 \mathrm{~h}$. All materials were presented on a computer monitor controlled by a Pentium II PC. The text appeared in white characters on a black background. Participants were instructed to place their right thumb on a line advance key, their right index finger on a "yes" key, and their left index finger on a "no" key. Each experimental session started with 50 lexical decision test items to allow the participants to practice with the response keys.

After the lexical decision practice, the participants were given the instructions for the experiment. They were instructed to read at a comfortable pace and to keep their fingers on the response keys at all times. The participants pressed the line advancement key after reading each line to move from one line to the next. Each press of the line advancement key erased the current line of text and presented the next line. The press of the line advancement key after the presentation of the discussion sentence initiated the recognition test word routine. First, the words "Get Ready" flashed on the screen for $500 \mathrm{msec}$, then the recognition test word appeared in the middle of the screen in all capital letters. The test word remained on the screen until the participant pressed either the "yes" or the "no" key. Participants were instructed to respond "yes" if the recognition test word had been presented earlier in the passage and "no" if the word had not appeared in the passage. When the participants responded correctly the words "Correct Response" flashed in the upper right-hand corner of the screen for $1,500 \mathrm{msec}$ and for incorrect responses the words "Incorrect Response" flashed in the same manner. Between the stories the word "Next" appeared at the bottom center of the screen. After every block of six stories, six true or false ("yes" or "no") comprehension statements were presented to encourage careful participation.

\section{Results and Discussion}

For Experiments 2-4, $F_{1}$ refers to tests against an error term based on subject variability and $F_{2}$ refers to tests against an error term based on item variability. Results reported as significant had associated $p$ values of less than .05. Outliers (response times that were more than $2 S D$ from the subject means) were discarded, resulting in a loss of less than .05 of the data in each experiment. Mean recognition times, error rates, and mean reading times for Experiment 2 are presented in Table 1.

Recognition probes. Overall, the pattern of results mirrored that in Experiment 1; participants responded to probe words in the two discussion conditions faster than

Table 1

Recognition Response Times, Error Rates, and Pre-Probe Reading Times (in Milliseconds) for Experiment 2

\begin{tabular}{lccc}
\hline & No Discussion & Discussion & Urgent Discussion \\
\hline Recognition & 751 & 715 & 707 \\
Error rate & .07 & .05 & .06 \\
Pre-probe reading & 1,846 & 1,900 & 1,868 \\
\hline
\end{tabular}


they did in the no-discussion condition, and the urgentdiscussion probes were slightly faster than the discussion probes. The discussion effect was significant $\left[F_{1}(2,84)=\right.$ $\left.7.49, M S_{\mathrm{e}}=2,415, F_{2}(2,66)=7.89, M S_{\mathrm{e}}=5,180\right]$. Followup comparisons revealed significant differences between the no-discussion control and both the discussion and the urgent-discussion conditions $\left[F_{1}(1,42)=11.68, M S_{\mathrm{e}}=\right.$ 2,$341 ; F_{2}(1,33)=6.43, M S_{\mathrm{e}}=6,170$, and $F_{1}(1,42)=10.35$, $M S_{\mathrm{e}}=2,602 ; F_{2}(1,33)=8.24, M S_{\mathrm{e}}=4,397$ for the discussion and urgent-discussion conditions, respectively]. The difference between the two discussion conditions was not significant (both $F_{\mathrm{S}}<1$ ).

Pre-probe sentences. One possible explanation of the probe response times is that reading times differed substantially among the sentences immediately preceding the probes, which necessarily differed in wording and syntax. If the no-discussion sentence was more difficult to comprehend, spillover processing could account for the slower responding to the probe in that condition. To test this possibility, we analyzed the reading times for the pre-probe sentences. Overall, the means were quite similar (Table 1) and, if anything, the pre-probe reading times were read faster-not slower-in the no-discussion passages. Comparisons of all three means produced no significant differences by subjects $\left(F_{1}<1\right)$; when tested against item variability, the no-discussion mean time was shorter than the two discussion means $\left[F_{2}(1,33)=7.75, M S_{\mathrm{e}}=60,502\right.$; $F_{2}(1,33)=14.69, M S_{\mathrm{e}}=71,516$, for no-discussion compared with the urgent-discussion and discussion conditions, respectively]. Thus spillover effects do not account for differences in response times to the probes.

We used the recognition-probe task to keep the present study as similar as possible to previous related research (Greene et al., 1994; Lea et al., 1998; McKoon et al., 1996). However, it has been argued that recognition responses may be affected by post-lexical-access processes such as context checking. According to this view, if the context immediately preceding the probe differs between conditionsand the pre-probe context in inference versions is more similar to the probe than it is in the control-then "yes" responses may be facilitated. In the present set of materials, context checking is unlikely because (1) the context near the time of test, if different, does not systematically favor the "higher activation" conditions; (2) measures not subject to context checking have been used to provide converging evidence (e.g., Lea et al., 1998, Experiment 3; Experiments 1 and 5 in the present study); and (3) the "inference" versions of these passages have led to significantly slower recognition times (e.g., Experiment 4). Therefore any context-checking account simply cannot explain the variety of results derived by past and present results using these materials with a recognition task.

In summary, previous experiments have demonstrated the reactivation of a backgrounded concept when the protagonist associated with that concept was reintroduced into the narrative. The results of the present experiment, together with those of Experiment 1, provide converging evidence of an elaborative inference when the two protagonists engaged in a conversation following the reunion.
Specifically, there was greater activation of the inferential concept in the discussion conditions than in the control.

\section{EXPERIMENT 3}

In Experiments 1 and 2, the reunion sentences in the nodiscussion condition could not be the same as the reunion sentences in the two discussion conditions. In the nodiscussion version, the protagonists did not interact during the reunion, although they were both mentioned in the sentence (e.g., one of them was sleeping). It is possible that the interactive reunion sentence ${ }^{2}$ was required for reactivation of the target concept. If so, any differences observed after the discussion sentences might reflect an effect that was due to the nature of the reunion, rather than to the discussion, sentences.

Experiment 3 tested this alternative explanation. Passages contained either the noninteractive or the interactive reunion sentence; these were the reunion sentences from the no-discussion and the discussion versions in Table A1. There were three probe conditions. The target concept probe was presented immediately before a reunion sentence, after the noninteractive reunion sentence, or after the interactive reunion sentence. If the type of reunion sentence was responsible for the effects previously observed, there should be no difference in response times to the probe as a function of whether it was placed before a reunion sentence or after the noninteractive reunion sentence, and both times should be slower than when the probe was placed after the interactive reunion sentence. Alternatively, if all that is required for reactivation of the target concept is the reinstatement of the protagonist associated with it (e.g., Jane), then response times after the reunion sentences should be equal and faster than the response time prior to a reunion. If this is the observed pattern, differences obtained following the no-discussion and discussion sentences would have to be attributed to the discussion condition and not to the reunion sentences preceding the discussion sentences.

Experiment 3 also provides a test of the resonance model proposed by Myers and O'Brien (1998; O'Brien \& Myers, 1999). That model would predict that the reunion manipulation in Experiment 3 should have no effect on the reactivation of backgrounded information because both the noninteractive and interactive reunion sentences provide a cue for reactivation, that cue being the reinstated character who is associated with the target concept. According to the resonance model, the mere mention of such a cue should be enough to reactivate associated backgrounded information; the difference between the interactive and noninteractive reunions should not influence this effect. However, if an effect of the type of reunion is found, the resonance model would be unable to explain the findings.

\section{Method}

Participants. Fifty-two Macalester College students participated in return for course credit. All participants were native speakers of English.

Materials and Procedure. The 42 passages used in Experiment 2 were used in Experiment 3 . The only difference was the place- 
ment of the recognition test words. One third of the probes were placed after the continuation section, immediately prior to the reunion sentence. Another one third of the probes were placed after the noninteractive reunion sentence, and the remaining third were placed after the interactive reunion sentence. Again, we attempted to equate the length of the pre-probe sentences. The mean pre-probe line length was $37.8,38.6$, and 38.2 characters for the before-reunion, after-noninteractive, and after-interactive passages, respectively. The procedure for Experiment 3 was the same as in Experiment 2.

\section{Results and Discussion}

Recognition probes. The recognition response times, accuracy, and pre-probe reading times for Experiment 3 are presented in Table 2 . The reunion effect reported in previous research (e.g., Lea et al., 1998) is replicated in the probe means. Specifically, the two after-reunion probe positions led to faster response times than in the before-reunion control. More relevant to the present study, both interactive and noninteractive reunions produced this effect.

The effect of the probe position was significant $\left[F_{1}(2,98)=3.91, M S_{\mathrm{e}}=2,518 ; F_{2}(2,66)=4.66, M S_{\mathrm{e}}=\right.$ $6,605]$. Follow-up comparisons revealed significant differences between responses after the noninteractive reunion and the before-reunion control $\left[F_{1}(1,49)=5.94\right.$, $\left.M S_{\mathrm{e}}=2,130 ; F_{2}(1,33)=5.14, M S_{\mathrm{e}}=7,218\right]$ and between responses following the interactive reunion versus the control $\left[F_{1}(1,49)=5.29, M S_{\mathrm{e}}=2,812 ; F_{2}(1,33)=8.42\right.$, $\left.M S_{\mathrm{e}}=6,382\right]$. The two postreunion means were not different from each other (both $F_{\mathrm{S}}<1$ ).

Pre-probe sentences. In Experiment 3 the pre-probe sentence was the reunion sentence in the two discussion conditions (e.g., Gloria was still awake when Jane arrived home) and the prereunion sentence (e.g., After dinner she put dishes in the dishwasher) in the control condition. As in Experiment 2, we examined these reading times to determine whether a spillover explanation of the probe response times was possible. For example, if the reading times for the before-reunion (control) condition were significantly slower than the interactive reunion times, the parallel result for the recognition probes could be explained by spillover processing. The means presented in Table 2 show a different trend: Reading times were slower for the noninteractive reunion sentence compared with either the prereunion or the interactive reunion sentence. This main effect for pre-probe sentence was significant $\left[F_{1}(2,98)=49.46, M S_{\mathrm{e}}=43,795 ; F_{2}(2,70)=10.47, M S_{\mathrm{e}}=\right.$ $145,106]$, as was the comparison between the noninteractive reunion sentence versus the before-reunion sentence $\left[F_{1}(1,49)=80.12, M S_{\mathrm{e}}=43,347 ; F_{2}(1,35)=12.34\right.$, $\left.M S_{\mathrm{e}}=201,588\right]$ and versus the interactive reunion sen-

Table 2

Recognition Response Times, Error Rates, and Pre-Probe Reading Times (in Milliseconds) for Experiment 3

\begin{tabular}{lccc}
\hline & $\begin{array}{c}\text { Before } \\
\text { Reunion }\end{array}$ & $\begin{array}{c}\text { After Noninteractive } \\
\text { Reunion }\end{array}$ & $\begin{array}{c}\text { After Interactive } \\
\text { Reunion }\end{array}$ \\
\hline Recognition & 773 & 752 & 745 \\
Error rate & .05 & .04 & .04 \\
Pre-probe reading & 1,964 & 2,322 & 1,971 \\
\hline
\end{tabular}

tence $\left[F_{1}(1,49)=63.16, M S_{\mathrm{e}}=47,631 ; F_{2}(1,35)=19.12\right.$, $\left.M S_{\mathrm{e}}=107,234\right]$. The pre-probe reading times for the interactive reunion and before-reunion sentences did not differ significantly (both $F_{\mathrm{S}}<1$ ). Thus, despite the fact that the pre-probe sentences were equated in length, the noninteractive reunion sentences produced, on average, significantly longer reading times. We have no ready explanation for this difference. Note, however, that this reading slowdown would tend to slow responses only following the noninteractive reunion sentence. Therefore, spillover effects cannot account for the faster probe times following the reunion sentences.

This experiment was designed to investigate whether or not the type of reunion influenced the reactivation of backgrounded information. Two results argue that reunion type does not make a difference: (1) The reunion effect (faster recognition times after the reunion than before) was obtained with both the interactive and noninteractive reunions; and (2) the two reunion types were not different from each other. This result rules out a reunion-type explanation for the results of Experiment 2. Therefore, the discussion effect found in Experiment 2 appears to be a result of the discussion manipulation, as we originally concluded.

The evidence that the noninteractive reunion sentence serves to reactivate the backgrounded concept is consistent with results reported by Albrecht and Myers (1995, 1998). In the early part of their passages, the protagonist had an important goal whose completion was interrupted. Later in the passage an action inconsistent with the goal caused a slowdown in reading, but only if an object was mentioned that had been last mentioned several sentences earlier in the context of the protagonist's goal. Albrecht and Myers hypothesized that the reinstatement of the object in the text served as a signal to memory, initiating a resonance process that resulted in the reactivation of the goal. Using different materials and a different response measure, Experiment 3 provided results consistent with those of Albrecht and Myers. Presumably, re-encountering Jane in the narrative resulted in reactivation of a concept that had been associated with her (her cousin). This process required only mentioning Jane and was not dependent on the potential interaction of Jane and Gloria.

\section{EXPERIMENT 4}

One way of viewing the discussion effects found in Experiments 1 and 2 is that a mechanism such as the constraint-satisfaction process in Kintsch's (1988) integration stage operates to integrate currently active text and world knowledge concepts and propositions. Assuming that the discussion sentence activates world knowledge about conversations, in the previous experiments the reactivated information was readily integrated with that knowledge; it essentially filled the topic slot in a conversational schema. But what if the targeted information had not been an appropriate topic for the discussion? In Experiment 4 , we included passages in which the target is related to a secret that one protagonist must keep from the 
other. When one protagonist has a secret but indicates to the other protagonist that she has something urgent to relate (e.g., "I have got to tell you something," said Jane), there are various possible consequences. If the inference is the result of a conscious constructive process, it may immediately be suppressed. In that case, there will be no difference in probe response times between the urgentdiscussion secret condition and the no-discussion secret condition. A second possibility is that the process will proceed just as in the conditions of Experiments 1 and 2, in which the topic was not confidential. In that case, the urgent-discussion secret condition will yield faster response times than the no-discussion secret control, as in Experiment 2. There is a third possibility. As O'Brien and Myers (1999; also see van den Broek, Risden, \& HusebyeHartmann, 1995) have noted, there must be a process that evaluates the outcome of the attempted integration. When this process signals a failure of comprehension, as when the topic should not be part of a conversation, the reader may consciously attempt to draw other inferences that will satisfy the constraints of the text. Either because that attempt has settled on some prediction inconsistent with the probe, or because the attentional resources involved in the attempt are not available for a response to the probe, responses in such situations may be slowed. In this view, response times to the probe in the urgent-discussion secret condition will be slow when one protagonist has a secret.

In summary, Experiment 4 involved the three conditions of Experiments 1 and 2 (which can be viewed as "nosecret" in the context of this experiment ) and three additional conditions in which one protagonist has a secret from the other. As we have discussed, there are grounds for three alternative outcomes with respect to the urgentdiscussion/secret response times. It is not clear whether the standard discussion/secret condition should have any effect.

\section{Method}

Participants. Eighty Macalester College students participated in Experiment 4 in return for $\$ 10$. All participants were native speakers of English.

Materials and Procedure. Thirty-six passages based on those used in Lea et al. (1998) were used in Experiment 4. Two versions of each story were created: a secret version and a no-secret version. These passages differed from those used in Experiment 2 in the introduction section and, often, in the probe word. In the secret condition, the two protagonists were introduced and then parted. The target character or concept was then introduced in the context of one of the protagonists. The secret was created between the target character and the protagonist and the text communicated that the secret should not be shared with the other protagonist. Table A2 presents an example passage in which, in the secret conditions, the secret was a surprise birthday party for Gloria. This secret was shared by the other protagonist, Jane, and Jane's cousin. In the no-secret condition, the target concept was a surprise birthday party for Jane's uncle. Again, it was shared by Jane and her cousin, but it was not a secret to be withheld from Gloria. The probe was the word the secret was about (e.g., party). We felt confident that concepts other than the outsider character (e.g., cousin) would be reactivated by the reunion sentence. Research has shown that entire goals can be reactivated from earlier parts of a story merely by adding a contextual cue that is not related a priori to the goal (e.g., Albrecht \& Myers, 1995). Other researchers using versions of the present passages have conducted a parametric series of experiments that nicely demonstrate the variety of concepts that can be reactivated from the introductory sections of these passages (McKoon et al., 1996).

There were three discussion conditions resulting in a 2 (secret/nosecret) $\times 3$ (discussion condition) within-subjects design. The preprobe lines were equated as much as possible. The mean pre-probe line lengths were $39.4,39.4$, and 39.5 characters for the no-discussion, discussion, and urgent-discussion passages, respectively. The procedure for Experiment 4 was the same as that described for Experiment 2.

\section{Results and Discussion}

Recognition probes. The recognition response times, accuracy, and pre-probe reading times for Experiment 4 are presented in Table 3. Response times in the no-secret condition followed a pattern similar to that found in Experiment 2 , with the two discussion means notably faster than the no-discussion control. When the target concept was a secret, however, a different pattern emerged in which recognition times in the urgent-discussion condition were slower than those in both the discussion and the no-discussion condition. This difference in pattern led to a significant discussion $\times$ secret interaction $\left[F_{1}(2,148)=5.76, M S_{\mathrm{e}}=\right.$ 5,$\left.992 ; F_{2}(2,58)=3.64, M S_{\mathrm{e}}=14,429\right]$. Follow-up tests revealed that in the no-secret condition, the no-discussion mean was significantly slower than the discussion mean by subjects $\left[F_{1}(1,74)=17.00, M S_{\mathrm{e}}=6,709\right]$ and marginally slower by items $\left[F_{2}(1,29)=3.81, M S_{\mathrm{e}}=12,227, p=\right.$ $.06]$. The no-discussion mean was significantly slower than the urgent-discussion mean $\left[F_{1}(1,74)=11.31, M S_{\mathrm{e}}=\right.$ 3,$\left.891 ; F_{2}(1,29)=5.64, M S_{\mathrm{e}}=8,668\right]$. In the secret condition, the urgent-discussion mean was significantly slower than the no-discussion mean $\left[F_{1}(1,74)=4.73, M S_{\mathrm{e}}=\right.$ 5,$\left.533 ; F_{2}(1,29)=5.70, M S_{\mathrm{e}}=9,254\right]$. The discussion versus no-discussion difference was significant by subjects $\left[F_{1}(1,74)=3.90, M S_{\mathrm{e}}=6,670\right]$ but not by items $\left(F_{2}<1\right)$, as was the discussion versus urgent-discussion difference $\left[F_{1}(1,74)=20.99, M S_{\mathrm{e}}=4,967 ; F_{2}(1,29)=1.60, M S_{\mathrm{e}}=\right.$ $14,011, p=.21]$.

The recognition probe times for the no-secret condition replicated the discussion effect found in Experiment 2. However, in the secret condition the recognition times did not replicate the discussion effect. The longer recognition times for the urgent-discussion/secret condition suggest that readers cognitively "stumble" when presented with the probe word that refers to the secret held from the protagonist. The source of the slower response times presumably lies in a failure to integrate the target concept into the con-

Table 3

Recognition Response Times, Error Rates, and Pre-Probe Reading Times (in Milliseconds) for Experiment 4

\begin{tabular}{lcccccccc}
\hline & \multicolumn{3}{c}{ No-Secret } & & \multicolumn{3}{c}{ Secret } \\
\cline { 2 - 4 } \cline { 6 - 8 } & No Dis & Dis & Urgent Dis & & No Dis & Dis & Urgent Dis \\
\hline Recognition & 796 & 746 & 764 & & 778 & 755 & 804 \\
Error rate & .07 & .09 & .07 & & .08 & .08 & .07 \\
Pre-probe & 1,636 & 1,644 & 1,629 & & 1,709 & 1,673 & 1,605 \\
\hline
\end{tabular}

Note-Dis, discussion. 
versational schema. As earlier studies (Greene et al., 1994; Lea et al., 1998) and Experiment 3 demonstrated, the target was reactivated when the associated protagonist was reinstated into the narrative. However, discussion of that target violates the secret. Consequently, responses are delayed because the reader is attempting to resolve the incompatibility of the inference with the knowledge that it involves a secret.

Pre-probe sentences. As in Experiments 2 and 3, the pattern of reading times in Experiment 4 is not consistent with a spillover interpretation of the recognition task data. In the no-secret condition, the variance of the three condition means was not significant by subjects $\left(F_{1}<1\right)$ or items $\left[F_{2}(2,54)=2.31, M S_{\mathrm{e}}=36,983, p=.11\right)$. In the secret conditions, the urgent-discussion sentences were read more quickly than the no-discussion sentences [significant by subjects, $F_{1}(1,72)=11.83, M S_{\mathrm{e}}=33,623$, and by items, $\left.F_{2}(1,27)=4.0, M S_{\mathrm{e}}=59,769\right]$ and read faster than the discussion sentences [significant by subjects, $F_{1}(1,72)=$ $4.91, M S_{\mathrm{e}}=26,211$, but not by items, $F_{2}(1,27)=4.0$, $\left.M S_{\mathrm{e}}=48,842, p=.27\right]$. In contrast to this pattern, a spillover interpretation of the recognition data would require that reading times be longest in the urgent-discussion condition.

\section{EXPERIMENT 5}

The secret/no-secret manipulation used in Experiment 4 led to clearly different patterns in response times across the three probe positions, as detected for example, by the significant secret $\times$ discussion interaction. Specifically, whereas the urgent-discussion sentences led to significantly faster recognition times when the target was not secret, they led to significantly slower recognition times when the target was a secret. This reversal did not generalize to the discussion condition in which the protagonists merely chatted for a while. Our predictions about the secret/ no-secret manipulation focused on the urgent-discussion condition; still, we were curious to know more about what was happening in the discussion condition. (The secret/ discussion response times were significantly faster than both no-discussion and urgent-discussion responses in Experiment 4, but only in the subjects analysis.)

In Experiment 5 we returned to the continuation-writing design of Experiment 1 in an attempt to flesh out readers' responses to the secret passages. Unlike our predictions for Experiment 1, those for this experiment did not involve the prediction that high availability would necessarily be associated with a high mention rate in the secret conditions. Indeed, the processes under investigation include those that evaluate the appropriateness of active concepts to the current discourse. Thus, we expected that participants would not mention the target (e.g., party) as often in the secret/discussion passages compared with the no-discussion control, despite its relatively high availability. The continuations for the urgent-discussion passages are more difficult to predict. On the one hand, it is intuitively reasonable to expect that readers would be even less likely to include the secret target-concept in an urgent-discussion continuation as they would be to include it in a discussion continuation. On the other hand, the urgent-discussion passages require readers to somehow square (1) a secret between one protagonist and the other with (2) the secretbaring protagonist saying, I've got to tell you something. In many of our passages the concept to be kept secret was something that in life-and especially in fiction-is "spilled" to someone who should not know. ${ }^{3}$ Furthermore, unlike the discussion passages in which the protagonists could "chat" about any number of topics presented earlier in the passage, there are no readily available topics for an urgent discussion; the secret concept is the only choice. Thus, in constructing a continuation for a story in which a secret-baring protagonist tells the protagonist-in-the-dark I need to tell you something, we thought that participants might steer the passage toward being a "spilling the beans" story, not a story about keeping a secret. Hence, mentions in the secret/urgent-discussion condition might well be relatively high compared with those in the other two secret conditions.

For the no-secret passages we expected results consistent with what we found in Experiment 1-more mentions following discussion sentences than following no-discussion sentences. One difference from Experiment 1 that we expected was a lower overall mention rate due to the presence of a second potential topic of discussion. For instance, in the example passage for Experiment 1, only the cousin was a likely topic of conversation; in the no-secret version in Experiment 5, both cousin and party were available to fill the discussion slot. Thus, we predicted an overall lower mention rate than in Experiment 1.

\section{Method}

Participants. Forty-four Macalester College students participated in Experiment 5 in return for course credit. All participants were native speakers of English.

Materials and Procedure. The 36 stories used in Experiment 4 were used in this experiment (see Table A2 for an example). The organization of the materials into stimulus lists and the procedure were the same as in Experiment 1.

\section{Results and Discussion}

The same two criteria used in Experiment 1 to operationally define a mention of the target concept were used in the present experiment. Thus, by the "reference" criterion, any unambiguous reference to the target concept was counted as a mention, and by the "nominal" criterion only proper names, social roles, or concepts referring specifically to the target (e.g., party) were counted as mentions. Two experimenters coded the participants' protocols using both the reference and nominal criteria. Once again, no statistical difference was found between the two mention categories, so the more inclusive reference criterion was used for analysis.

Participants read 36 passages in six conditions, so there were six passages per participant per condition. The proportions of mention are presented in Table 4 . The pattern among the discussion levels was different between the secret and no-secret conditions; this led to a significant 
Table 4

Proportion of Target Concept Mentions in Experiment 5

\begin{tabular}{ccccccc}
\hline \multicolumn{3}{c}{ No Secret } & & \multicolumn{3}{c}{ Secret } \\
\cline { 1 - 2 } \cline { 5 - 7 } No Dis & Dis & Urgent Dis & & No Dis & Dis & Urgent Dis \\
\hline .37 & .60 & .65 & & .35 & .26 & .42 \\
\hline
\end{tabular}

secret $\times$ discussion interaction, just as we found in Experiment $4\left[F(2,76)=21.32, M S_{\mathrm{e}}=1.023\right]$.

For the three passage types in the secret condition, the target was mentioned in the no-discussion control, on average, 2.11 times in the 6 opportunities to do so (35\%), 1.58 times in the discussion condition $(26 \%)$, and 2.52 times in the urgent-discussion condition (42\%). Thus, the urgent-discussion passages led to the most mentions, followed by the no-discussion, and finally the discussion passages. This discussion effect among the three means was significant $\left[F(2,76)=10.08, M S_{\mathrm{e}}=.769\right]$. Follow-up two-tailed $t$ tests showed that the proportion of mentions in the discussion condition was significantly smaller than in the no-discussion condition [ $t(43)=2.29, S E M=.2287]$. The urgent-discussion versus the no-discussion comparison was not significantly different $[t(43)=1.55, S E M=$ .2648]. Participants on average mentioned the target protagonist more after urgent-discussion passages than after discussion passages $[t(43)=4.25, S E M=.4160]$. Thus, there were significantly fewer mentions in the discussion condition than in either of the other two conditions, which were not different from each other.

The pattern of mentions among the no-secret passages was similar to that found in Experiment 1 . That is, the three passage types were different as a set $[F(2,76)=33.72$, $\left.M S_{\mathrm{e}}=1.149\right]$, and both discussion and urgent-discussion passages led to more mentions than did the no-discussion control $[t(43)=5.08, S E M=.2640$, and $t(43)=5.86$, SEM $=$ .2833 for the discussion and urgent-discussion passages, respectively]. The proportions of mentions for the two discussion conditions were not significantly different from each other $[t(43)=1.14, S E M=.2793, p=.26]$.

In sum, the finding in the no-secret passages replicated the results from Experiment 1, even though the passages had been changed somewhat (a new concept was introduced, such as a party, and the target concept that counted as a mention was different). The secret passages led to a different pattern in which participants mentioned the target concept significantly less often when writing continuations for discussion passages compared with either the urgent-discussion or the no-discussion passages.

These results accord well with our predictions. First, we expected the no-secret stories to replicate the results of Experiment 1, but with an overall lower level of mention. We predicted a lower mention rate because changes made to the passages to accommodate the secret manipulation led to more discussion-topic possibilities.

Our predictions for the secret passages reflected a combination of the participants' understanding of the social conventions of secret keeping as well as their sophistica- tion in predicting outcomes based on the interaction of two pieces of information. That is, we thought that the combination of a secret/urgent-discussion sentence such as Boy, have I got something to tell you! might constitute a sort of coherence break that continuation writers would need to resolve, perhaps by resolving the secret between the two protagonists. By this reasoning, participants would mention the secret concept more often following urgentdiscussion sentences than following plain discussion sentences, in which the combination of a secret and "chatting" led to no such interaction. This pattern is essentially what we found in the secret passages in Experiment 5. The combination of a secret and a discussion sentence led to a significant reduction of mentions of the secret concept, whereas the secret/urgent-discussion combination led to no reduction of mentions and, if anything, a (nonsignificant) $7 \%$ increase of mentions over the no-discussion control. The two discussion conditions were reliably different, with urgent-discussion sentences leading to significantly more mentions than did discussion sentences.

These data provide a perspective from which to view the activation results for secret conditions in Experiment 4. First, it appears that the easy availability of other topics appropriate for a "chatting" discussion may have preempted any need to inhibit the secret concept. This possibility is consistent with secret/discussion response times in Experiment 4 that were not significantly slowed and the significantly lower target mentions in that condition of Experiment 5, as well as an overall lowering of mentions in the no-secret passages relative to Experiment 1. Conversely, the lack of conversation-topic alternatives for an urgent discussion might both decrease activation of the secret and lead readers to resolve the awkwardness by making the story about revealing-rather than keeping-a secret. By this view, we would expect low availability immediately after the discussion sentence and a relatively high proportion of mention. The results from Experiments 4 and 5 accord well with this account.

\section{GENERAL DISCUSSION}

The results of these experiments reflect three sequential and collaborative mechanisms involved in comprehension. The first is the activation, or reactivation, of concepts and propositions in the representation of the discourse. Several previous studies have demonstrated that the text currently in focus provides cues that serve to activate related knowledge, through either argument overlap or semantic associations (e.g., Greene et al., 1994; Lea et al., 1998; McKoon et al., 1996). In those studies, one protagonist was reinstated into the text and engaged the second protagonist in conversation. The results of Experiment 3 of the present study extend the previous findings by demonstrating that the mere mention of a previously backgrounded protagonist is sufficient to reactivate a concept associated with that individual; interaction between the two protagonists is not necessary for reactivation. Myers 
and O'Brien (1998) have hypothesized that such results are the consequence of a passive resonance process. Their article provides a detailed model of this process, together with the results of computer simulations.

The second mechanism, reflected in the results of Experiments 1 and 2, is integration of text information with world knowledge. We assume that sentences introducing a discussion between the two protagonists activate schematic knowledge about conversations. That schema requires discussants and a topic. In the discussion condition, the topic is filled by the target concept that has been reactivated by the reintroduction of the protagonist with which it has been associated. Kintsch's (1988) integration stage provides one possible explanation of how the target concept becomes associated with the conversational schema. Once the target is integrated with the protagonists into the reader's conversational schema, its activation level will likely be maintained. The urgent-discussion condition may be explained in the same way with the added assumption that such sentences are stronger cues for the activation of the conversational schema. Alternatively, sentences such as $I$ have something to tell you may evoke a more conscious and effortful process in which the reader actively seeks to anticipate what the "something" is. In the no-discussion condition, there is no basis for integrating the target concept with the propositions in the sentence (Gloria was asleep when Jane arrived home) and therefore the activation level decays.

The third mechanism, reflected in the results of Experiments 4 and 5, is evaluation. Readers usually know when something fails to make sense. In the secret/urgentdiscussion condition of Experiment 4, the results of the integration of the target (e.g., party) into the conversation are presumably evaluated in the context of other reactivated information. Such other information would include the fact that the party is to be kept a secret from Gloria; that is, in the context of their conversation it is not an appropriate topic. This may cause the reader to devote attentional resources to search for an alternative topic or may even cause the reader to anticipate an alternative. In either case, when the probe appears, the reader will not be prepared for it.

Experiment 5 afforded a glance at how readers may cope with texts that raise flags in the evaluation process. With the secret/urgent-discussion texts, readers faced a potential anomaly: The protagonist who is guarding a secret from the other protagonist has just told that person that he/she has something he/she wants to discuss urgently. In the absence of other easily available urgent topics, many participants chose to take the story in a direction that preserves coherence: They made the story about one protagonist revealing a secret to the other. When faced with the ordinary discussion sentence, the evaluation process raised no flags because there were sufficient alternative topics for the situation, including "small-talk" topics (e.g., the weather) with which we are all too familiar.

Although it is clear that some evaluatory process underlies the monitoring of our comprehension, the nature of that process is unclear. One possibility is that it is an extension of the integration stage. In the secret condition of Experiment 4, not only is party reactivated, but so also is the knowledge that the party is to be a secret from Gloria. McKoon et al. (1996) have provided evidence that the reactivation process involves several interrelated concepts and propositions. Although party may be integrated into the topic of the conversation, the proposition that Jane will tell Gloria about the party must also be integrated with the proposition that the party is a secret, and with our world knowledge about the meaning of a secret. That integration process fails in the secret/urgent-discussion condition of Experiment 4. Therefore, by this account, success at integration is tantamount to evaluation.

Another possible extension of the integration stage that further fleshes out the evaluation process emerges from Keysar, Barr, Balin, and Paek's (1998) perspectiveadjustment model. The perspective-adjustmentmodel was developed to specify when and how common ground is considered during comprehension. The model assumes that two processes occur: (1) A fast, unrestricted search that interprets, for example, a definite reference with no regard to common ground, and (2) a monitoring and adjustment process that is sensitive to considerations of common ground (Keysar et al., 1998, p. 16). Although the perspective-adjustment model was introduced and tested in the context of research about the role of common ground in comprehension, it may well apply to the elaborative inferences under consideration in Experiment 4. By this account, after the discussion sentence the reader's quick, unrestricted search for a topic of conversation leads him/her to the target (e.g., party) until the adjustment phase can make the correction (in the secret condition) that the target is a poor fit, thereby creating a delay in the recognition times. Further research would be needed to test the validity of this account. In principle, methodologies such as those used by Keysar and his colleagues that interrupt processing to provide a processing "snap shot" (e.g., Horton \& Keysar, 1996) might be used to elucidate the details of the integration/evaluation stage.

The present research may also be seen as relevant to recent work by Gerrig, Brennan, and Ohaeri (2001). Gerrig et al. investigated how readers project knowledge to protagonists by using passages in which one protagonist has privileged information about some event that a second protagonist does not have. Gerrig et al. found that readers reliably used utterances in the texts to project knowledge to the intended protagonist(s). Thus, readers (1) were able to appreciate differences between their own knowledge and that of the characters in the stories, and (2) relied upon the characters' utterances as evidence to make inferences about who-knows-what-about-whom. Result 1 is consistent with Lea et al.'s (1998) finding (Experiment 4) that readers slow down on the pronoun sentence when it refers to a person that is not part of the protagonists' common ground (see the standard conclusion in Table A1). Result 2 is relevant to the present work; using Gerrig et al.'s terms, 
readers appreciated that the target concept (cousin, party) was privileged information, and that sentences like They chatted for a while projected information about the topic of conversation. Thus, the elaborative inferences studied here may constitute a special case of the way readers project knowledge as described by Gerrig et al.

In summary, there has been considerable skepticism about the extent of elaborative inferences during reading (e.g., Graesser et al., 1994; McKoon \& Ratcliff, 1992). Results such as those obtained by Lea (1995) with logical inferences, and the present results, indicate that such inferences may occur more frequently than was previously thought, and generated via mechanisms different from those usually invoked in this literature. This is not to suggest that elaborative inferences are ubiquitous. Indeed, our position is largely consistent with earlier statements about elaborative inferences. We assume that such inferences will be drawn when two conditions are met: (1) Necessary discourse elements are active, either because they have just been explicitly stated or because they have been reactivated by appropriate cues in the text; and (2) those discourse elements are strongly related to propositions or schematic knowledge that the reader brings to the text.

The present research builds upon work by others in which schematic knowledge is brought to bear on the comprehension process, and in doing so this work further establishes the role of more constructive, integrative processes in the formulation of elaborative inferences. Importantly, we think, the elaborative inferences under consideration in this paper were made possible by low-level, memory-based text processes such as resonance. Thus, previous work that investigated how memory-based processes contribute to comprehension (e.g., Lea et al., 1998; McKoon et al., 1996) can be viewed as further specifying the construction phase, whereas the present work may be seen as elaborating upon how information activated by such bottom-up processes can be recruited to make expectationdriven predictive inferences.

Finally, it is worth noting that by using the same passages that were used in the memory-based work, we are in a position now to complete the circle and explain how Marilyn, Jane's cousin, went from activation due to explicit mention early in the story, to reactivation via the reunion sentence and resonance, to being the predictable and inferrable topic of a discussion by way of schematic knowledge. As models of reading are further developed, we anticipate that bottom-up and top-down processes will be further reunited in ways that reflect their true deep collaboration in everyday comprehension.

\section{REFERENCES}

Albrecht, J. E., \& Myers, J. L. (1995). The role of context in accessing distant information during reading. Journal of Experimental Psychology: Learning, Memory, \& Cognition, 21, 1459-1468.

Albrecht, J. E., \& Myers, J. L. (1998). Accessing distant text information during reading: Effects of contextual cues. Discourse Processes, 26, 87-107.

Calvo, M. G., \& Castillo, M. D. (1996). Predictive inferences occur on-line, but with delay: Convergence of naming and reading times. Discourse Processes, 22, 57-78.

Calvo, M. G., Castillo, M. D., \& Estevez, A. (1999). On-line predictive inferences in reading: Processing time during versus after the priming context. Memory \& Cognition, 27, 834-843.

Estevez, A., \& Calvo, M. G. (2000). Working memory capacity and time course of predictive inferences. Memory, 8, 51-61.

GARROD, S., \& SANFORD, A. (1977). Interpreting anaphoric relations: The integration of semantic information while reading. Journal of Verbal Learning \& Verbal Behavior, 16, 77-90.

Gerrig, R. J., Brennan, S. E., \& Ohaeri, O. J. (2001). What characters know: Projected knowledge and projected co-presence. Journal of Memory \& Language, 44, 81-95.

Graesser, A. C., Singer, M., \& Trabasso, T. (1994). Constructing inferences during narrative text comprehension. Psychological Review, 101, 371-395.

Greene, S. B., Gerrig, R. J., McKoon, G., \& Ratcliff, R. (1994). Unheralded pronouns and management by common ground. Journal of Memory \& Language, 33, 511-526.

Haviland, S. E., \& Clark, H. H. (1974). What's new? Acquiring new information as a process of comprehension. Journal of Verbal Learning \& Verbal Behavior, 13, 512-521.

Horton, W. S., \& Keysar, B. (1996). When do speakers take into account common ground? Cognition, 59, 91-117.

Keefe, D. E., \& McDaniel, M. A. (1993). The time course and durability of predictive inferences. Journal of Memory \& Language, 32, 446-463.

Keenan, J. M., Baillet, S. D., \& Brown, P. (1984). The effects of causal cohesion comprehension and memory. Journal of Verbal Learning \& Verbal Behavior, 23, 115-126.

Keysar, B., Barr, D. J., Balin, J. A., \& Paek, T. (1998). Definite reference and mutual knowledge: Process models of common ground in comprehension. Journal of Memory \& Language, 39, 1-20.

Kintsch, W. (1988). The role of knowledge in discourse comprehension: A construction-integration model. Psychological Review, 95, 163-182.

Klin, C. M., Murray, J. D., Levine, W. H., \& Guzman, A. E. (1999). Forward inferences: From activation to long-term memory. Discourse Processes, 27, 241-260.

LEA, R. B. (1995). On-line evidence for elaborative logical inferences in text. Journal of Experimental Psychology: Learning, Memory, \& Cognition, 21, 1469-1482.

Lea, R. B., Mason, R. A., Albrecht, J. E., Birch, S. L., \& Myers, J. L. (1998). Who knows what about whom: What role does common ground play in accessing distant information? Journal of Memory \& Language, 39, 70-84.

Lea, R. B., \& Mulligan, E. J. (2002). The effect of negation on deductive inferences. Journal of Experimental Psychology: Learning, Memory, \& Cognition, 28, 303-317.

McKoon, G., Gerrig, R. J., \& Greene, S. B. (1996). Pronoun resolution without pronouns: Some consequences of memory-based text processing. Journal of Experimental Psychology: Learning, Memory, \& Cognition, 22, 919-932.

McKoon, G., \& RATCLIFF, R. (1980). The comprehension processes and memory structures involved in anaphoric reference. Journal of Verbal Learning \& Verbal Behavior, 19, 668-682.

McKoon, G., \& Ratcliff, R. (1986). Inferences about predictable events. Journal of Experimental Psychology: Learning, Memory, \& Cognition, 12, 82-91.

McKoon, G., \& Ratcliff, R. (1992). Inference during reading. Psychological Review, 99, 440-466.

McKoon, G., \& Ratcliff, R. (1995). The minimalist hypothesis: Directions for research. In C. A. Weaver III, S. Mannes, \& C. R. Fletcher (Eds.), Discourse comprehension: Essays in honor of Walter Kintsch (pp. 97-116). Hillsdale, NJ: Erlbaum.

Murray, J. D., Klin, C. M., \& Myers, J. L. (1993). Forward inferences in narrative text. Journal of Memory \& Language, 32, 464-473.

Myers, J. L., \& O'Brien, E. J. (1998). Accessing the discourse representation during reading. Discourse Processes, 26, 131-157.

Myers, J. L., Shinjo, M., \& Duffy, S. A. (1987). Degree of causal 
relatedness and memory. Journal of Memory \& Language, 4, 453465.

O'Brien, E. J., Duffy, S. A., \& Myers, J. L. (1986). Anaphoric inference during reading. Journal of Experimental Psychology: Learning, Memory, \& Cognition, 12, 346-352.

O’Brien, E. J., \& Myers, J. L. (1999). Text comprehension: A view from the bottom up. In S. R. Goldman, A. C. Graesser, \& P. van den Broek (Eds.), Narrative comprehension, causality, \& coherence (pp. 3553). Mahwah, NJ: Erlbaum.

Potts, G. R., Keenan, J. M., \& Golding, J. M. (1988). Assessing the occurrence of elaborative inferences: Lexical versus naming. Journal of Memory \& Language, 27, 399-415.

Singer, M. (1979). Processes of inference during sentence encoding. Memory \& Cognition, 7, 192-200.

Singer, M., \& Ferreira, F. (1983). Inferring consequences in story comprehension. Journal of Verbal Learning \& Verbal Behavior, 22, 437-448. van den Broek, P., Risden, K., \& Husebye-Hartmann, E. (1995). The role of readers' standards for coherence in the generation of inferences during reading. In R. F. Lorch, Jr. \& E. J. O’Brien (Eds.), Sources of coherence in reading (pp. 353-373). Hillsdale, NJ: Erlbaum.

\section{NOTES}

1. As Potts et al. (1988) have stated, "There is no uniformly accepted criterion for what it means to "draw an inference"' (p. 405). Following their lead, we will regard activation of a concept central to the inference as evidence of the inference, provided there is no basis for assuming that the activation is based on lexical associations.

2. Although we refer to this sentence (e.g., Gloria was still awake when Jane arrived home) as "interactive," it actually only establishes a potential for interaction as opposed to the noninteractive reunion sentence in which Gloria is asleep.

3. We thank an anonymous reviewer for suggesting this possibility.

\section{APPENDIX \\ Example Text \\ Table A1 \\ Example Story From Lea et al. (1998, Standard Conclusion) and From Experiments 1 and 2 (No-Discussion, Discussion, and Urgent-Discussion Conclusions)}

Introduction

Jane and her roommate Gloria were leaving work. "Are you headed home?" asked Gloria. "No, but I'll see you later on tonight," replied Jane. Jane drove off to have dinner with her cousin, Marilyn. As she drove, she started to have regrets. She usually got sick when she ate at her cousin's. Jane wondered why she had agreed to go. She decided she was just too wimpy to say no.

\section{Continuation}

Meanwhile, Gloria went home and decided to cook something nice for herself. "As long as I'm home alone," she thought, "I'll eat well." Gloria searched her refrigerator for ingredients. She found enough eggs to make a quiche. After dinner, she put the dishes in the dishwater.

\section{Standard Conclusion}

Reunion sentence:

Recognition test word:

Concluding sentence:

Concluding sentence:

Reunion sentence: Discussion sentence: Recognition test word: Concluding sentence: Concluding sentence:

\section{Reunion sentence:}

Discussion sentence: Recognition test word: Concluding sentence: Concluding sentence:

Reunion sentence: Discussion sentence: Recognition test word: Concluding sentence: Concluding sentence:
Gloria was still up when Jane arrived home about midnight. COUSIN Gloria asked Jane, "Did she play you old disco records?" Jane chuckled and said, "I can't get Disco Inferno out of my mind."

\section{No-Discussion Conclusion}

Gloria was sound asleep when Jane arrived home. Jane tiptoed around so as not to wake Gloria. COUSIN

"She must have had a relaxing evening," thought Jane.

"I think I'll go to bed now too," she decided.

\section{Discussion Conclusion}

Gloria was still awake when Jane arrived home.

They chatted together for a couple of minutes.

COUSIN

Gloria asked Jane, "Did she play you old disco records?"

Jane chuckled and said, "I can't get Disco Inferno out of my mind."

Urgent-Discussion Conclusion

Gloria was still awake when Jane arrived home.

"I have got to tell you something," said Jane.

COUSIN

Gloria asked Jane, "Did she play you old disco records?"

Jane chuckled and said, "I can't get Disco Inferno out of my mind." 
APPENDIX (Continued)

\section{Table A2 \\ Example Text From Experiment 4}

Introduction

Secret

Jane and her roommate, Gloria, were leaving work. "Are you headed home?" asked Gloria. "No, but I'll see you later tonight," replied Jane. Jane drove off to have dinner with her cousin, Marilyn. They were going to plan a surprise birthday party for Gloria. Tomorrow was Gloria's 25th birthday and Jane was very excited about surprising her.

\section{No-Secret}

Jane and her roommate, Gloria, were leaving work. "Are you headed home?" asked Gloria. "No, but I'll see you later tonight," replied Jane. Jane drove off to have dinner with her cousin, Marilyn. They were going to plan a surprise birthday party for Jane's uncle. Tomorrow was his 50th birthday and Jane was very excited about surprising him.

\section{Continuation}

Meanwhile, Gloria went home and decided to cook something nice for herself. "As long as I'm home alone," she thought, "I'll eat well." Gloria searched the refrigerator for ingredients. She found enough eggs to make a quiche. After dinner, she put the dishes in the dishwater.

\section{No-Discussion Conclusion}

Reunion sentence:

Discussion sentence:

Recognition test word:

Concluding sentence:

Concluding sentence:

Reunion sentence: Discussion sentence: Recognition test word: Concluding sentence: Concluding sentence:

Reunion sentence: Discussion sentence: Recognition test word: Concluding sentence: Concluding sentence:
Gloria was sound asleep when Jane arrived home. Jane tiptoed around so as not to wake Gloria. PARTY

"She must have had a relaxing evening," thought Jane.

"I think I'll go to bed now too," she decided.

\section{Discussion Conclusion}

Gloria was still awake when Jane arrived home.

They chatted together for a couple of minutes.

PARTY

Gloria asked Jane, "Did she play you old disco records?"

Jane chuckled and said, "I can't get Disco Inferno out of my mind."

Urgent-Discussion Conclusion

Gloria was still awake when Jane arrived home.

"I have got to tell you something," said Jane.

PARTY

Gloria asked Jane, "Did she play you old disco records?"

Jane chuckled and said, "I can't get Disco Inferno out of my mind." 